\title{
The Modified Glasgow Prognostic Score as a Predictor of Survival After Hepatectomy for Colorectal Liver Metastases
}

\author{
Kazuya Nakagawa, $\mathrm{MD}^{1}$, Kuniya Tanaka, MD, $\mathrm{PhD}^{1}$, Kazunori Nojiri, MD, $\mathrm{PhD}^{1}$, Takafumi Kumamoto, MD, \\ $\mathbf{P h D}^{1}$, Kazuhisa Takeda, $\mathrm{MD}, \mathrm{PhD}^{1}$, Michio Ueda, $\mathrm{MD}, \mathrm{PhD}^{2}$, and Itaru Endo, $\mathrm{MD}, \mathbf{P h D}^{1}$ \\ ${ }^{1}$ Department of Gastroenterological Surgery, Yokohama City University Graduate School of Medicine, Yokohama, Japan; \\ ${ }^{2}$ Department of Surgery, Gastroenterological Center, Yokohama City University, Yokohama, Japan
}

\begin{abstract}
Background. The inflammation-based Glasgow prognostic score (GPS) has been demonstrated to be prognostic for various tumors. We investigated the value of the modified GPS (mGPS) for the prognosis of patients undergoing curative resection for colorectal liver metastases (CRLM). Methods. A total of 343 patients were enrolled onto this study. The mGPS was calculated as follows: mGPS-0, C-reactive protein $(\mathrm{CRP}) \leq 10 \mathrm{mg} / \mathrm{L} ; \mathrm{mGPS}-1$, CRP $>10 \mathrm{mg} / \mathrm{L}$ and albumin $\geq 35 \mathrm{~g} / \mathrm{L} ;$ and $\mathrm{mGPS}-2$, CRP $>10 \mathrm{mg} / \mathrm{L}$ and albumin $<35 \mathrm{~g} / \mathrm{L}$. Prognostic significance was retrospectively analyzed by univariate and multivariate analyses.

Results. Of the 343 patients, $295(86.0 \%)$ were assigned to mGPS-0, $33(9.6 \%)$ to mGPS-1, and 15 (4.4\%) to mGPS-2. The median disease-free survival of patients with mGPS- $0,-1$, and -2 was $18.3,15.5$, and 5.2 months, respectively. The median cancer-specific survival (CSS) of patients with mGPS-0, -1 , and -2 was $89.5,62.2$, and 25.8 months, respectively. The CSS of patients with mGPS-0 was significantly longer than that of patients with mGPS-2. Multivariate analysis revealed a significant association between cancer-related postoperative mortality and mGPS and carcinoembryonic antigen level.

Conclusions. The preoperative mGPS is a useful prognostic factor for postoperative survival in patients undergoing curative resection for CRLM.
\end{abstract}

(C) The Author(s) 2014. This article is published with open access at Springerlink.com

First Received: 5 May 2013;

Published Online: 23 January 2014

K. Nakagawa, MD

e-mail: nakagawakazuya0827@yahoo.co.jp
Colorectal cancer (CRC) is the third most common cancer worldwide, with a cumulative lifetime risk of $\sim 5 \%{ }^{1,2}$ Despite improvements in hepatectomy techniques (e.g., aggressive liver resection including major vessel resection and two-stage hepatectomy combined with chemotherapy) and introduction of new postoperative chemotherapy regimens, overall survival is still poor for most patients with colorectal liver metastases (CRLM). ${ }^{3-6}$ 5 -Year survival rates after hepatic resection reportedly range from 33 to $61 \% .^{7-13}$

There is increasing evidence that the presence of an ongoing systemic inflammatory response, as revealed by an elevated concentration of circulating serum $\mathrm{C}$-reactive protein (CRP), is associated with poor outcomes in patients with advanced cancers. ${ }^{14-20}$ Recent studies have revealed that the Glasgow prognostic score (GPS), an inflammation-based prognostic score that includes only serum CRP and serum albumin, is one of the most useful scoring systems for the prognostication of patients with advanced cancer. ${ }^{21-28}$ Several studies have investigated the value of the GPS for postoperative prognostication of patients undergoing curative resection for CRC. ${ }^{29,30}$ However, few studies have reported the GPS in patients with CRLM who underwent liver resection. ${ }^{31,32}$

The GPS was recently modified on the basis of evidence that hypoalbuminemia in patients without an elevated CRP concentration has no significant association with cancer-specific survival (CSS) ${ }^{30}$ There is a considerable body of evidence supporting that the modified GPS (mGPS) can predict CSS in patients undergoing curative resection for CRC. ${ }^{33-35}$ To our knowledge, no study has investigated the usefulness of the mGPS in patients undergoing liver resection for CRLM. Therefore, the aim of this study was to evaluate the value of the mGPS for prediction of postoperative death in patients with CRLM. 


\section{MATERIALS AND METHODS}

\section{Patients}

Between January 1988 and December 2010, a total of 433 patients with CRLM underwent initial liver resection at university-affiliated hospitals (Graduate School of Medicine, Yokohama City University, and Yokohama City University Medical Center). Of these, 75 were excluded from analysis because extrahepatic disease was present at surgery, 14 were excluded because data on their CRP and albumin levels were not available, and 1 was excluded because of postoperative death (within 30 days). The remaining 343 patients were enrolled onto this study. None of the patients exhibited clinical evidence of infection or any other inflammatory conditions. The median follow-up period for survivors was 54.4 months (range 2-237 months). The mGPS was estimated as described previously. ${ }^{33}$ Briefly, patients with an elevated CRP level $(>10 \mathrm{mg} / \mathrm{L}$ ) were allocated as mGPS- 1 or -2 depending on the absence or presence of hypoalbuminemia $(<35 \mathrm{~g} / \mathrm{L})$, and patients with no elevation of CRP $(\leq 10 \mathrm{mg} / \mathrm{L})$ were allocated as mGPS- 0 . The extent of the resection was recorded as major or minor, with a major resection defined as a resection of more than three segments.

\section{Patient Follow-Up}

Patients were followed up monthly at our outpatient clinic. Data were obtained and recorded, and long-term outcomes were determined through clinical follow-up, cancer registry follow-up, and contact with the patient, family, or referring physician when necessary. Serum CEA levels were measured monthly, and computed tomography (CT) was performed every 3 months. Recurrence was defined as a lesion that was biopsy-proven recurrent adenocarcinoma or a lesion that was deemed suspicious on cross-sectional imaging in the setting of an elevated CEA level. The end of follow-up was the time of last follow-up (March 2012) or death.

\section{Adjuvant Therapy}

After resection of liver metastases or extrahepatic metastases, adjuvant chemotherapy was carried out generally via hepatic artery infusion or by intravenous infusion, usually with 5-fluorouracil and 1-folinic acid with or without the addition of oxaliplatin or irinotecan. In all patients who received prehepatectomy chemotherapy, the same preoperative chemotherapy was usually continued postoperatively as adjuvant therapy.
Statistical Analysis

Data are presented as mean \pm standard error (SE). Differences between groups were analyzed using the Mann-Whitney $U$ test and $\chi^{2}$ test. Hazard ratios (HR) with $95 \%$ confidence intervals $(95 \% \mathrm{CI})$ were calculated using univariate or multivariate analysis.

Survival was measured from the time of hepatic resection to death, which was the end point. Survival curves were constructed by the Kaplan-Meier method and compared using a log-rank test. Statistical significance was defied as $P<0.05$. Analysis was performed using the software package Dr. SPSS II (SPSS Inc., Chicago, IL, USA).

\section{RESULTS}

\section{Relationships Between $m$ GPS and Patient Characteristics}

Among the 343 patients, $48(14.0 \%)$ had an elevated CRP level (>10 g/L) and $29(8.5 \%)$ had hypoalbuminemia $(<35 \mathrm{~g} / \mathrm{L})$. Fifteen $(4.4 \%)$ patients had both elevated CRP and hypoalbuminemia. Of the 343 patients, 295 (86.0\%) were assigned to mGPS-0, 33 (9.6\%) to mGPS-1, and 15 (4.4\%) to mGPS-2.

Patient characteristics in each mGPS group are provided in Table 1. Patients with mGPS-2 were older than those with mGPS-0 $(P=0.046)$ or $1(P=0.036)$. The maximum tumor size was larger in patients with mGPS-1 $(P=0.006)$ or $2(P=0.030)$ than in those with mGPS- 0 . Patients with mGPS- 1 or 2 had higher levels of white blood cells (mGPS-0 vs. $1, P=0.001 ;$ mGPS-0 vs. 2, $P<0.001$ ), neutrophils (mGPS- 0 vs. $1, P<0.001$; mGPS0 vs. $2, P<0.001$ ), and CEA (mGPS-0 vs. $1, P=0.036$; mGPS-0 vs. $2, P=0.005)$ than those of patients with mGPS-0. There were no significant differences between mGPS and patient characteristics such as gender, primary site (colon/rectum), timing (synchronous/metachronous), distribution (unilobar/bilobar), or tumor number.

\section{Recurrence and Recurrence Pattern}

Overall, $224(65.3 \%)$ patients experienced recurrence of disease at the time of last follow-up. The number of patients with recurrence in the mGPS- $0,-1$, and -2 groups was 190 of $295(64.4 \%), 22$ of $33(66.7 \%)$, and 12 of 15 $(80.0 \%)$, respectively. The median DFS of patients with mGPS-0, -1 , and -2 was $18.3,15.5$, and 5.2 months, respectively. Kaplan-Meier analysis and the log-rank test demonstrated no significant differences among patients with mGPS-0, -1 , and $-2(P=0.2125)$ (Fig. 1). 
TABLE 1 Relationship between mGPS and clinical background

\begin{tabular}{|c|c|c|c|c|c|c|}
\hline Characteristic & $\begin{array}{l}\text { mGPS-0 } \\
(n=295)\end{array}$ & $\begin{array}{l}P \text { value, mGPS- } 0 \\
\text { vs. } 1\end{array}$ & $\begin{array}{l}\text { mGPS-1 } \\
(n=33)\end{array}$ & $\begin{array}{l}P \text { value, mGPS- } 1 \\
\text { vs. } 2\end{array}$ & $\begin{array}{l}\text { mGPS-2 } \\
(n=15)\end{array}$ & $\begin{array}{l}P \text { value, mGPS- } 0 \\
\text { vs. } 2\end{array}$ \\
\hline Age & $63.7 \pm 0.6$ & 0.495 & $52.0 \pm 1.7$ & 0.036 & $69.5 \pm 2.7$ & 0.046 \\
\hline \multicolumn{7}{|l|}{ Gender } \\
\hline Male & $191(65 \%)$ & 0.826 & $22(67 \%)$ & 0.082 & $6(40 \%)$ & 0.052 \\
\hline Female & $104(35 \%)$ & & $11(33 \%)$ & & $9(60 \%)$ & \\
\hline \multicolumn{7}{|l|}{ Site of primary lesion } \\
\hline Colon & $166(57 \%)$ & 0.953 & $19(58 \%)$ & 0.482 & $7(47 \%)$ & 0.429 \\
\hline Rectum & $125(43 \%)$ & & $14(42 \%)$ & & $8(53 \%)$ & \\
\hline \multicolumn{7}{|l|}{$\begin{array}{l}\text { Histologic } \\
\quad \text { differentiation }\end{array}$} \\
\hline Moderate & $179(63 \%)$ & 0.020 & $14(42 \%)$ & 0.119 & $10(67 \%)$ & 0.789 \\
\hline Other & $104(37 \%)$ & & $19(58 \%)$ & & $5(33 \%)$ & \\
\hline \multicolumn{7}{|l|}{ Timing } \\
\hline Synchronous & $144(49 \%)$ & 0.052 & $22(67 \%)$ & 0.746 & $11(63 \%)$ & 0.110 \\
\hline Metachronous & $151(51 \%)$ & & $11(33 \%)$ & & $4(27 \%)$ & \\
\hline \multicolumn{7}{|l|}{ Distribution } \\
\hline Unilobar & $178(60 \%)$ & 0.328 & 17. $(52 \%)$ & 0.907 & $8(53 \%)$ & 0.589 \\
\hline Bilobar & $177(40 \%)$ & & $16(48 \%)$ & & $7(47 \%)$ & \\
\hline No. of liver metastases & $3.3 \pm 0.2$ & 0.094 & $5.5 \pm 1.1$ & 0.262 & $3.5 \pm 1.3$ & 0.960 \\
\hline Single & $131(44 \%)$ & 0.582 & $13(39 \%)$ & 0.636 & $7(47 \%)$ & \\
\hline Multiple & $164(56 \%)$ & & $20(61 \%)$ & & $8(53 \%)$ & 0.864 \\
\hline $\begin{array}{l}\text { Maximum tumor size, } \\
\mathrm{cm}\end{array}$ & $33.3 \pm 1.4$ & 0.006 & $47.9 \pm 5.5$ & 0.954 & $48.5 \pm 8.0$ & 0.030 \\
\hline \multicolumn{7}{|l|}{ Extent of liver resection } \\
\hline Minor & $202(68 \%)$ & $<0.001$ & $12(36 \%)$ & 0.053 & $10(67 \%)$ & 0.883 \\
\hline Major & $93(32 \%)$ & & $21(64 \%)$ & & $5(33 \%)$ & \\
\hline \multicolumn{7}{|l|}{$\begin{array}{l}\text { Perioperative } \\
\text { chemotherapy }\end{array}$} \\
\hline Neo-adjuvant & $62(21 \%)$ & 0.979 & $7(21 \%)$ & 0.056 & 0 & 0.047 \\
\hline \multicolumn{7}{|l|}{ Adjuvant } \\
\hline Systemic & $60(20 \%)$ & & $7(21 \%)$ & & $1(7 \%)$ & \\
\hline HAI & $75(25 \%)$ & 0.679 & $11(33 \%)$ & 0.202 & $6(40 \%)$ & 0.284 \\
\hline Systemic + HAI & $49(17 \%)$ & & $4(12 \%)$ & & $3(20 \%)$ & \\
\hline \multicolumn{7}{|l|}{$\begin{array}{l}\text { Preoperative laboratory } \\
\text { data }\end{array}$} \\
\hline White blood cell & $5,600 \pm 99$ & 0.001 & $6,900 \pm 402$ & 0.081 & $7,900 \pm 528$ & $<0.001$ \\
\hline Neutrophil & $3,300 \pm 84$ & $<0.001$ & $4,400 \pm 331$ & 0.052 & $5,300 \pm 403$ & $<0.001$ \\
\hline Lymphocyte & $1,600 \pm 35$ & 0.684 & $1,600 \pm 125$ & 0.584 & $1,432 \pm 117$ & 0.280 \\
\hline CEA, ng/L & $88 \pm 22$ & 0.036 & $316 \pm 160$ & 0.268 & $418 \pm 228$ & 0.005 \\
\hline
\end{tabular}

$m G P S$ modified Glasgow prognostic score, $H A I$ hepatic arterial infusion, $C E A$ carcinoembryonic antigen

The initial recurrence pattern is summarized in Table 2. The initial recurrence pattern was liver only in $84(24.5 \%)$ patients, lung only in $61(17.8 \%)$, other single sites in 43 $(12.5 \%)$, and multiple organ sites in 34 (9.9\%). There were no significant differences in the initial recurrence pattern between mGPS- $0,-1$, and $-2(P=0.126)$. We focused on the initial liver-only recurrence, and the numbers of recurrent tumors among the mGPS groups are summarized in Table 3.
The number of patients with liver-only recurrence in the mGPS-0, - 1, and - 2 groups was 72, 8, and 4, respectively. The number of patients with multiple liver recurrences $(\geq 4)$ in the mGPS-0, -1, and -2 groups was 19 (26.4\%), 3 (37.5\%), and $4(100 \%)$, respectively. The number of patients with multiple liver recurrences in the mGPS-0 group (19 of 72) was significantly smaller than that in the mGPS-1 and 2 groups (7 of $12 ; P=0.03$ ). 
FIG. 1 Relationship between mGPS $(0,1$, and 2 from top to bottom) and disease-free survival in patients with CRLM (a). Relationship between mGPS $(0,1$, and 2 from top to bottom) and cancer-specific survival in patients with CRLM (b)

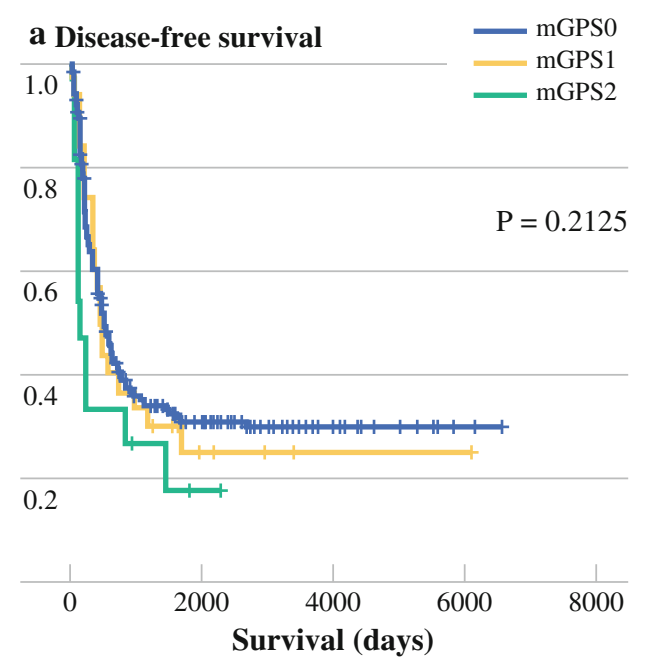

TABLE 2 Relationship between mGPS and initial recurrence pattern

\begin{tabular}{llll}
\hline Site of recurrence & $\begin{array}{l}\text { mGPS-0 } \\
(n=190)\end{array}$ & $\begin{array}{l}\text { mGPS-1 } \\
(n=22)\end{array}$ & $\begin{array}{l}\text { mGPS-2 } \\
(n=12)\end{array}$ \\
\hline Liver only & $72(38 \%)$ & $8(36 \%)$ & $4(33 \%)$ \\
Lung only & $50(26 \%)$ & $8(36 \%)$ & $3(25 \%)$ \\
Other single sites & $36(19 \%)$ & $3(14 \%)$ & $4(33 \%)$ \\
Multiple organ sites & $30(16 \%)$ & $3(14 \%)$ & $1(9 \%)$ \\
Unknown & $2(1 \%)$ & 0 & 0 \\
\hline
\end{tabular}

mGPS modified Glasgow prognostic score

\section{Resection for Liver or Lung Recurrence}

Among the 84 patients with liver recurrence, 35 (41.7\%) underwent resection. Resection for initial liver recurrence was performed in $34(47.2 \%)$ patients with mGPS-0, in no patients with mGPS-1, and in $1(25.0 \%)$ patient with mGPS-2 (Table 3). The rate of resected liver recurrence was significantly higher in patients with mGPS0 than in those with mGPS-1 and $2(P<0.01)$. Radiofrequency ablation (RFA) was performed in two (2.8\%) patients with mGPS-0, in two $(25.0 \%)$ with mGPS-1, and in none with mGPS-2. The rate of treatment for local control, including liver resection and RFA, was also significantly higher in patients with mGPS-0 than in those with mGPS- 1 and $2(P<0.01)$.

On the other hand, among 82 patients with lung recurrence, $27(32.9 \%)$ underwent resection. Resection for initial lung recurrence was performed in $22(31.9 \%)$ patients with mGPS-0, in $5(55.6 \%)$ with mGPS-1, and in none with mGPS-2. The rate of resected lung recurrence tended to be higher in patients with mGPS-0 or 1 than in those with mGPS-2, but there was no significant difference among the three groups $(P=0.133)$.
TABLE 3 Relationship between mGPS and number of tumor recurrences in patients with initial liver-only recurrence

\begin{tabular}{llll}
\hline No. of tumor recurrences & $\begin{array}{l}\text { mGPS-0 } \\
(n=72)\end{array}$ & $\begin{array}{l}\text { mGPS-1 } \\
(n=8)\end{array}$ & $\begin{array}{l}\text { mGPS-2 } \\
(n=4)\end{array}$ \\
\hline 1 & $31(43 \%)$ & $3(37.5 \%)$ & 0 \\
2 & $13(18 \%)$ & 0 & 0 \\
3 & $9(13 \%)$ & $2(25 \%)$ & 0 \\
$\leq 4$ & $19(26 \%)$ & $3(37.5 \%)$ & $4(100 \%)$ \\
Resection & $34(47 \%)$ & 0 & $1(25 \%)$ \\
\hline
\end{tabular}

$m G P S$ modified Glasgow prognostic score

Survival

Overall, $94(27.4 \%)$ patients died during this study. Of these, there were eight non-cancer-related deaths. The median CSS of patients with mGPS-0, -1 , and -2 was 89.5 , 62.2, and 25.8 months, respectively. Kaplan-Meier analysis and the log-rank test demonstrated significant differences among patients with mGPS-0, -1, and -2 $(P=0.0044)$, with a higher cancer-related mortality rate in patients with a higher mGPS (Fig. 1). Kaplan-Meier analysis and the log-rank test demonstrated that patients with mGPS-0 survived longer than those with mGPS-2 $(P=0.0040)$. Furthermore, survival of patients with mGPS-1 tended to be poorer than that of patients with mGPS-0 ( $P=0.0584)$.

\section{Prognostic Factors}

Univariate analysis of postoperative mortality is listed in Table 4. Excluding treatment-related factors, ten factors were included in the univariate analysis. Six factors were significantly associated with CSS including timing, 
TABLE 4 Univariate and multivariate analysis in relation to cancer-specific death

\begin{tabular}{|c|c|c|c|c|c|c|}
\hline \multirow[t]{2}{*}{ Variable } & \multicolumn{3}{|c|}{ Univariate analysis } & \multicolumn{3}{|c|}{ Multivariate analysis } \\
\hline & Hazard ratio & $95 \% \mathrm{CI}$ & $P$ value & Hazard ratio & $95 \% \mathrm{CI}$ & $P$ value \\
\hline Age $(<65 / \geq 65$ years $)$ & 1.026 & $0.736-1.430$ & 0.879 & - & - & - \\
\hline Gender (F/M) & 0.884 & $0.627-1.246$ & 0.482 & - & - & - \\
\hline Site of primary lesion (colon/rectum) & 1.042 & 0.7461 .456 & 0.809 & - & - & - \\
\hline Timing (metachronousmeta/synchronous) & 1.585 & $1.130-2.224$ & 0.008 & 1.296 & $0.875-1.921$ & 0.196 \\
\hline Distribution (unilobular/bilobular) & 1.647 & $1.182-2.296$ & $<0.001$ & 0.951 & $0.584-1.551$ & 0.842 \\
\hline No. of tumors $(<2 / \geq 2)$ & 1.788 & $1.260-2.537$ & 0.001 & 1.528 & $0.933-2.504$ & 0.092 \\
\hline Maximum tumor size $(<3 / \geq 3 \mathrm{~cm})$ & 1.496 & $1.069-2.093$ & 0.019 & 1.038 & $0.693-1.557$ & 0.855 \\
\hline Pathologic differentiation (well differentiated/other) & 0.982 & $0.691-1.394$ & 0.917 & - & - & - \\
\hline CEA $(<30 / \geq 30 \mathrm{ng} / \mathrm{L})$ & 2.117 & $1.498-2.991$ & $<0.001$ & 2.044 & $1366-3.058$ & 0.001 \\
\hline mGPS $(0 / 1 / 2)$ & 1.611 & $1.202-2.159$ & 0.001 & 1.595 & $1.156-2.201$ & 0.004 \\
\hline
\end{tabular}

$C I$ confidence interval, $C E A$ carcinoembryonic antigen, $m G P S$ modified Glasgow prognostic score

distribution, number, maximum tumor size, mGPS, and CEA. On multivariate analysis, factors with a $P$ value of $<0.05$ in the univariate analysis were included. Multivariate analysis revealed a significant association between cancer-related postoperative mortality and mGPS (HR $1.595 ; 95 \%$ CI $1.156-2.201 ; P=0.004)$ and CEA (HR 2.044; $95 \%$ CI 1.366-3.058; $P=0.001)$.

\section{DISCUSSION}

The present retrospective study analyzed individual clinical data for 343 patients who underwent hepatectomy among a pure cohort of patients with CRLM. Our results demonstrate the prognostic value of the mGPS for CRLM. Multivariate analysis revealed a significant association between CSS and mGPS and CEA. A few studies have evaluated the GPS in patients undergoing liver resection for CRLM; however, to our knowledge, this is the first study to investigate the usefulness of the mGPS. There was no difference in DFS among patients with mGPS- $0,-1$, and -2 $(P=0.2125)$, and patients with mGPS-0 had a smaller number of liver recurrences, resulting in a much lower rate of recurrence resection than in patients with mGPS- 1 and 2.

Many studies have reported that elevated CRP levels are indicative of a poor outcome in a variety of cancers. ${ }^{16-18}$ For example, Wong et al. ${ }^{20}$ reported that an elevated preoperative CRP is a predictor of poor outcome in patients undergoing curative resection for CRLM Ishizuka et al. ${ }^{31}$ reported that in multivariate analysis, elevated CRP levels as well as the number of metastatic tumors were associated with cancer-specific death. On the other hand, hypoalbuminemia is often observed in patients with advanced cancer and is usually regarded as a good index for malnutrition and cachexia. In patients with colorectal cancer, hypoalbuminemia was reported to be associated with a poorer outcome. ${ }^{30,36}$
The GPS, which is based on both serum elevation of CRP and hypoalbuminemia, may enable a better appreciation of effects of the tumor on both ongoing systemic inflammation and malnutrition. The GPS was introduced to predict the prognosis of patients with advanced neoplasms. $^{21,25,29,30,37}$ The GPS was recently modified on the basis of evidence that hypoalbuminemia in patients without an elevated CRP concentration has no significant association with $\mathrm{CSS}^{30}$

At present, only two studies have investigated the usefulness of the GPS for postoperative death of patients undergoing curative resection for CRLM. One study revealed that in multivariate analysis, a GPS of one or two and three or more liver metastases were independent prognostic factors among 63 patients. ${ }^{32}$ The other study revealed that the GPS was able to classify 93 patients with resectable CRLM into three independent groups and that GPS was associated with postoperative cancer-related death in univariate analysis, but not in multivariate analysis. ${ }^{31}$ To date, most series on the value of GPS for patients undergoing liver resection for CRLM have been limited by small sample sizes. The sample size of the present study was relatively large. Our results revealed that a higher mGPS was associated with poorer survival in patients undergoing liver resection for CRLM, which is accordance with results of previous studies evaluating the prognostic value of the mGPS in colorectal and other cancers.

Although there were no significant differences between mGPS and timing (synchronous/metachronous), there seemed to be a trend toward higher mGPS in the synchronous group. These may influence aggressive biological behavior of synchronous liver metastases.

The clinical risk score (CRS), a score from 0 to 5 based on five preoperative variables, had been developed as useful prognostic scoring system and this system has been 
validated at other institutions. ${ }^{38-40}$ In our series, CRS high ( $\geq 3$ ) was not significantly associated with CSS in univariate analysis (HR 0.809; $95 \%$ CI 0.506-1.294; $P=0.377)$. Neoadjuvant chemotherapy was administered to $69(20.1 \%)$ patients and chemotherapy has changed some variables such as tumor size and CEA. Therefore, our study might not demonstrate the usefulness of the CRS.

Although our results demonstrated the prognostic value of the mGPS, Kaplan-Meier analysis and the log-rank test demonstrated no differences in DFS among patients with mGPS-0, -1 , and $-2(P=0.2125)$. Two previously reported studies did not investigate the relationship between the GPS and DFS. Several recent studies on the pattern of recurrence after hepatectomy have been reported. Hill et al. $^{41}$ reported that patients with liver-only or lung-only recurrence had a better prognosis compared with patients with other patterns of recurrence. D'Angelica et al. ${ }^{42}$ reported that the recurrence pattern and resection of the recurrence were independently associated with survival from the time of recurrence in multivariate analysis. Patients with recurrence in multiple sites reportedly had a shorter survival time than patients with recurrence in a single site; furthermore, patients with resected recurrences, regardless of site, survived longer than those who did not undergo resection. In our series, recurrence patterns exhibited no differences among patients with mGPS-0, 1, or 2. Patients with mGPS-0 had much less liver recurrence and underwent much fewer recurrence resections than did patients with mGPS-1 and 2. These differences in the patterns of recurrence and proportion of resections of recurrence according to the mGPS may affect CSS.

Patients with mGPS-1 and 2 have an elevated CRP level. An elevated CRP level may be indicative of a favorable environment for the establishment and growth of distant metastases. ${ }^{20}$ In cell culture experiments, CRP was found to inhibit apoptosis of carcinoma cells, thereby directly regulating tumor cell growth and survival. ${ }^{43}$ The serum level of vascular endothelial growth factor, an angiogenic factor, is increased in the presence of an elevated CRP level. ${ }^{44}$ Angiogenesis plays an important role in tumor growth and is associated with a poor outcome in patients with gastrointestinal tumors. ${ }^{45,46}$ An elevated CRP level may accelerate tumor aggressiveness and reflect the tumor recurrence pattern. Therefore, patients with mGPS-1 and 2 may develop recurrences of multiple liver metastases in the remnant liver.

It is known that liver resection is the only treatment that can be used to cure CRLM. ${ }^{47,48}$ Although the 5-year survival for patients with mGPS-2 was more than $40 \%$, these patients survived shorter than those with mGPS-0 $(P=0.0040)$. The number of patients with mGPS-2 was relatively small and no patient had received neoadjuvant chemotherapy. We think that the mGPS may be useful to select patients with poor prognosis. Although further study will be needed, additional treatment such as neoadjuvant or adjuvant chemotherapy or immunonutritional support may be indicated for patients with mGPS-2.

Potential limitations of the present study are that was retrospective in nature and involved one university-affiliated hospital. There were significant differences between mGPS and patient characteristics such as age, maximum tumor size, and preoperative CEA level. Therefore, a largescale prospective validation study is needed to confirm the results.

In conclusion, our results revealed that a higher mGPS was associated with multiple aggressive patterns of liver recurrence and poorer survival in patients with CRLM. The preoperative mGPS is a simple and useful prognostic factor for postoperative survival in patients undergoing liver resection for CRLM.

DISCLOSURE The authors declare no potential financial conflict of interest related to this manuscript.

OPEN ACCESS This article is distributed under the terms of the Creative Commons Attribution License which permits any use, distribution, and reproduction in any medium, provided the original author(s) and the source are credited.

\section{REFERENCES}

1. Rudy DR, Zdon MJ. Update on colorectal cancer. Am Fam Physician. 2000;61:1759-70, 1773-4.

2. Howe HL, Wu X, Ries LA, et al. Annual report to the nation on the status of cancer, 1975-2003, featuring cancer among US Hispanic/Latino populations. Cancer. 2006;107:1711-42.

3. Kawasaki S, Makuuchi M, Kakazu T, et al. Resection for multiple metastatic liver tumors after portal embolization. Surgery. 1994;115:674-7.

4. Adam R, Delvart V, Pascal G, et al. Rescue surgery for unresectable colorectal liver metastases downstaged by chemotherapy: a model to predict long-term survival. Ann Surg. 2004;240:644-57.

5. Tanaka K, Matsuyama R, Takeda K, Matsuo K, Nagano Y, Endo I. Aggressive liver resection including major-vessel resection for colorectal liver metastases. World J Hepatol. 2009;1:79-89.

6. Tanaka K, Kumamoto T, Nojiri K, Takeda K, Ichikawa Y, Endo I. Timing of two-stage liver resection during chemotherapy for otherwise unresectable colorectal metastases. World J Surg. 2012;36:1832-41.

7. Pawlik TM, Scoggins CR, Zorzi D, et al. Effect of surgical margin status on survival and site of recurrence after hepatic resection for colorectal metastases. Ann Surg. 2005;241:715-22.

8. Tanaka K, Shimada H, Ueda M, Matsuo K, Endo I, Togo S. Role of hepatectomy in treating multiple bilobar colorectal cancer metastases. Surgery. 2008;143:259-70.

9. de Haas RJ, Wicherts DA, Flores E, Azoulay D, Castaing D, Adam R. R1 resection by necessity for colorectal liver metastases: is it still a contraindication to surgery? Ann Surg. 2008;248:626-37.

10. Adam R, Wicherts DA, de Haas RJ, et al. Patients with initially unresectable colorectal liver metastases: is there a possibility of cure? J Clin Oncol. 2009;27:1829-35. 
11. Giuliante F, Ardito F, Vellone M, et al. Role of the surgeon as a variable in long-term survival after liver resection for colorectal metastases. J Surg Oncol. 2009;100:538-45.

12. de Jong MC, Pulitano C, Ribero D, et al. Rates and patterns of recurrence following curative intent surgery for colorectal liver metastasis: an international multi-institutional analysis of 1,669 patients. Ann Surg. 2009;250:440-8.

13. Muratore A, Ribero D, Zimmitti G, Mellano A, Langella S, Capussotti L. Resection margin and recurrence-free survival after liver resection of colorectal metastases. Ann Surg Oncol. 2010;17:1324-9.

14. Nozoe T, Matsumata T, Kitamura M, Sugimachi K. Significance of preoperative elevation of serum C-reactive protein as an indicator for prognosis in colorectal cancer. Am J Surg. 1998;176:335-8.

15. McMillan DC, Canna K, McArdle CS. Systemic inflammatory response predicts survival following curative resection of colorectal cancer. Br J Surg. 2003;90:215-9.

16. Koike Y, Miki C, Okugawa Y, et al. Preoperative C-reactive protein as a prognostic and therapeutic marker for colorectal cancer. J Surg Oncol. 2008;98:540-4.

17. Jagdev SP, Gregory W, Vasudev NS, et al. Improving the accuracy of preoperative survival prediction in renal cell carcinoma with C-reactive protein. Br J Cancer. 2010;103:1649-56.

18. Roxburgh CS, McMillan DC. Role of systemic inflammatory response in predicting survival in patients with primary operable cancer. Future Oncol. 2010;6:149-63.

19. Nozoe T, Saeki H, Sugimachi K. Significance of preoperative elevation of serum C-reactive protein as an indicator of prognosis in esophageal carcinoma. Am J Surg. 2001;182:197-201.

20. Wong VK, Malik HZ, Hamady ZZ, et al. C-reactive protein as a predictor of prognosis following curative resection for colorectal liver metastases. Br J Cancer. 2007;96:222-5.

21. Forrest LM, McMillan DC, McArdle CS, Angerson WJ, Dunlop DJ. Comparison of an inflammation-based prognostic score (GPS) with performance status (ECOG) in patients receiving platinum-based chemotherapy for inoperable non-small-cell lung cancer. Br J Cancer. 2004;90:1704-6.

22. Forrest LM, McMillan DC, McArdle CS, et al. A prospective longitudinal study of performance status, an inflammation-based score (GPS) and survival in patients with inoperable non-smallcell lung cancer. Br J Cancer. 2005;92:1834-6.

23. Elahi MM, McMillan DC, McArdle CS, Angerson WJ, Sattar N. Score based on hypoalbuminemia and elevated C-reactive protein predicts survival in patients with advanced gastrointestinal cancer. Nutr Cancer. 2004;48:171-3.

24. Brown DJ, Milroy R, Preston R, McMillan DC. The relationship between an inflammation based prognositic score (GPS) and changes in serum biochemical variables in patients with advanced lung and gastrointestinal cancer. J Clin Pathol. 2007;60:705-8.

25. Crumley AB, McMillan DC, McKernan M, McDonald AC, Stuart RC. Evaluation of an inflammation-based prognostic score in patients with inoperable gastro-oesophageal cancer. Br J Cancer. 2006;94:637-41.

26. Glen P, Jamieson NB, McMillan DC, Carter R, Imrie CW, McKay CJ. Evaluation of an inflammation-based prognostic score in patients with inoperable pancreatic cancer. Pancreatology. 2006;6:450-3.

27. Read JA, Choy ST, Beale PJ, Clarke SJ. Evaluation of nutritional and inflammatory status of advanced colorectal cancer patients and its correlation with survival. Nutr Cancer. 2006;55:78-85.

28. Ramsey S, Lamb GW, Aitchison M, Graham J, McMillan DC. Evaluation of an inflammation-based prognostic score in patients with metastatic renal cancer. Cancer. 2007;109:205-12.

29. Ishizuka M, Nagata $H$, Takagi $K$, Kubota $K$. Influence of inflammation-based prognostic score on mortality of patients undergoing chemotherapy for far advanced or recurrent unresectable colorectal cancer. Ann Surg. 2009;250:268-72.

30. McMillan DC, Crozier JE, Canna K, Angerson WJ, McArdle CS. Evaluation of an inflammation-based prognostic score (GPS) in patients undergoing resection for colon and rectal cancer. Int $J$ Colorectal Dis. 2007;22:881-6.

31. Ishizuka M, Kita J, Shimoda M, et al. Systemic inflammatory response predicts postoperative outcome in patients with liver metastases from colorectal cancer. $J$ Surg Oncol. 2009;100:38-42.

32. Kobayashi T, Teruya M, Kishiki T, et al. Elevated C-reactive protein and hypoalbuminemia measured before resection of colorectal liver metastases predict postoperative survival. Dig Surg. 2010;27:285-90.

33. Crozier JE, Leitch EF, McKee RF, Anderson JH, Horgan PG, McMillan DC. Relationship between emergency presentation, systemic inflammatory response, and cancer-specific survival in patients undergoing potentially curative surgery for colon cancer. Am J Surg. 2009;197:544-9.

34. Richards CH, Leitch EF, Horgan PG, Anderson JH, McKee RF, McMillan DC. The relationship between patient physiology, the systemic inflammatory response and survival in patients undergoing curative resection of colorectal cancer. $\mathrm{Br} J$ Cancer. 2010;103:1356-61.

35. Roxburgh CS, Platt JJ, Leitch EF, Kinsella J, Horgan PG, McMillan DC. Relationship between preoperative comorbidity, systemic inflammatory response, and survival in patients undergoing curative resection for colorectal cancer. Ann Surg Oncol. 2011;18:997-1005.

36. Lai CC, You JF, Yeh CY, et al. Low preoperative serum albumin in colon cancer: a risk factor for poor outcome. Int J Colorectal Dis. 2011;26:473-81.

37. Canna K, McArdle PA, McMillan DC, et al. The relationship between tumour T-lymphocyte infiltration, the systemic inflammatory response and survival in patients undergoing curative resection for colorectal cancer. Br J Cancer. 2005;92:651-4.

38. Fong Y, Fortner J, Sun RL, Brennan MF, Blumgart LH. Clinical score for predicting recurrence after hepatic resection for metastatic colorectal cancer: analysis of 1001 consecutive cases. Ann Surg. 1999;230:309-18.

39. Mala T, Bohler G, Mathisen O, Bergan A, Soreide O. Hepatic resection for colorectal metastases: can preoperative scoring predict patient outcome? World J Surg. 2002;26:1348-53.

40. Mann CD, Metcalfe MS, Leopardi LN, Maddern GJ. The clinical risk score: emerging as a reliable preoperative prognostic index in hepatectomy for colorectal metastases. Arch Surg. 2004;139:1168-72.

41. Hill CR, Chagpar RB, Callender GG, et al. Recurrence following hepatectomy for metastatic colorectal cancer: development of a model that predicts patterns of recurrence and survival. Ann Surg Oncol. 2012;19:139-44.

42. D'Angelica M, Kornprat P, Gonen M, et al. Effect on outcome of recurrence patterns after hepatectomy for colorectal metastases. Ann Surg Oncol. 2011;18:1096-103.

43. Yang J, Wezeman M, Zhang X, et al. Human C-reactive protein binds activating Fcgamma receptors and protects myeloma tumor cells from apoptosis. Cancer Cell. 2007;12:252-65.

44. Xavier P, Belo L, Beires J, et al. Serum levels of VEGF and TNFalpha and their association with C-reactive protein in patients with endometriosis. Arch Gynecol Obstet. 2006;273:227-31.

45. Tanigawa N, Amaya N, Matsumura M, Shimomatsuya T. Correlation between expression of vascular endothelial growth factor and tumor vascularity, and patient outcome in human gastric carcinoma. J Clin Oncol. 1997;15:826-32.

46. Fondevila C, Metges JP, Fuster J, et al. p53 and VEGF expression are independent predictors of tumour recurrence and survival 
following curative resection of gastric cancer. $\mathrm{Br} J$ Cancer. 2004;90:206-15.

47. Registry of hepatic metastases. Resection of the liver for colorectal carcinoma metastases: a multi-institutional study of indications for resection. Surgery. 1988;103:278-88.
48. Heslin MJ, Medina-Franco H, Parker M, Vickers SM, Aldrete J, Urist MM. Colorectal hepatic metastases: resection, local ablation, and hepatic artery infusion pump are associated with prolonged survival. Arch Surg. 2001;136:318-23. 